\title{
Anoperineal Tuberculosis in an HIV Patient
}

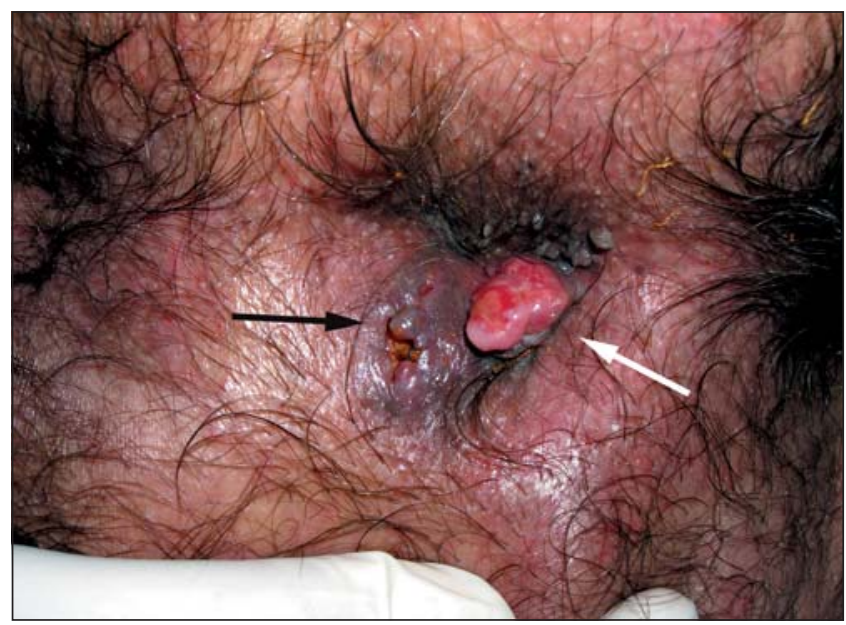

Figure 1. Ulcerated polipoid structure protruding through the anus (white arrow) and perianal fistula with purulent discharge (black arrow).
This is a case of a previously healthy 40-year old Caucasian male patient presenting on admission with progressive anal ulcerative lesion and perianal fistula in the last two months with purulent discharge, painful bowel movements and no blood in stool or diarrhea. He has had a recent diagnosis of HIV and Hepatitis C infection, without further medical work up to date. He denied loss of weight, sweating, fever, anorexia, abdominal pain, dysphagia, cough or any other respiratory symptoms. He was a current tobacco smoker and revealed a past history of abuse in alcohol and intravenous drugs. He also denied having any form of tuberculosis diagnosed in the past.

Laboratory studies showed normal values of haemoglobin, haematocrit, total leukocyte count, platelets and erythrocyte sedimentation rate. Liver enzymes and renal function tests were also within the normal range. His chest $\mathrm{x}$-ray was normal.

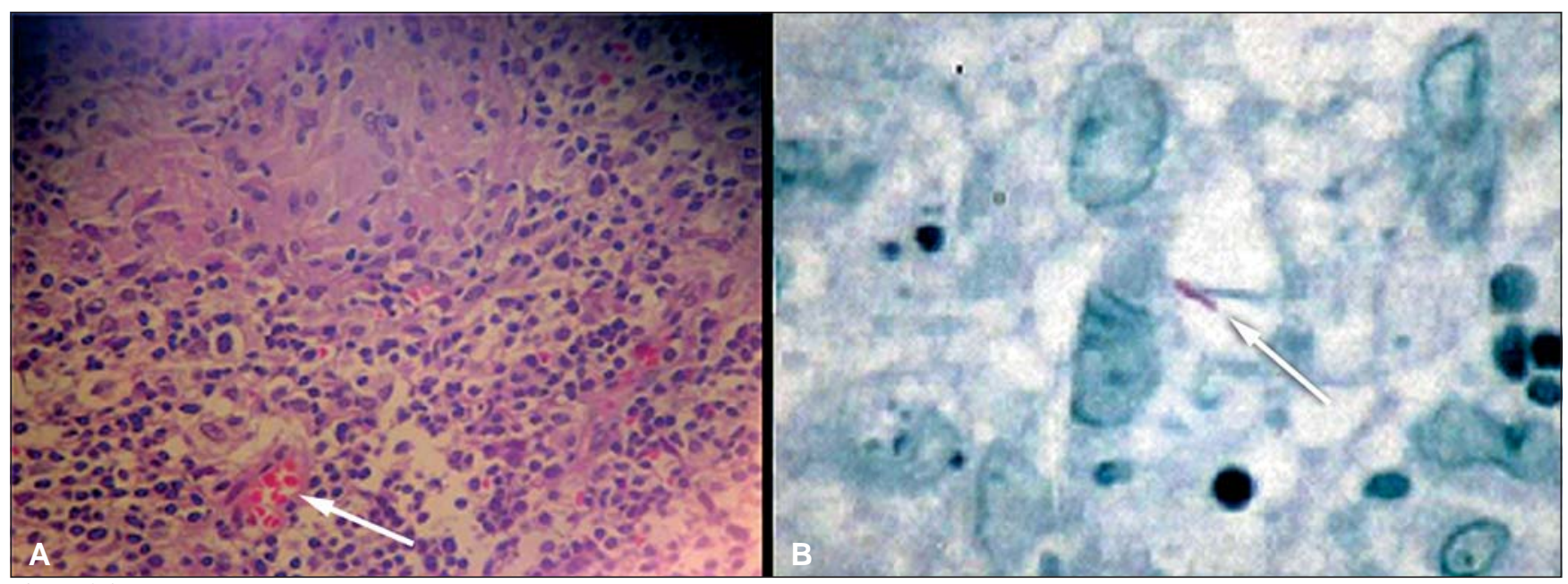

Figure 2.A- Hematoxylin/eosine stain of tissue showing granuloma with necrosis (400x). B- Ziehl-Nielsen stain preparation showing acid-fast bacillus (x400).

The physical examination showed no lymphadenopathy, and no palpable mass or organomegaly were detected on abdominal examination. Anoperineal examination showed an ulcerated polipoid structure protruding through the anus and a perianal fistula with purulent discharge (Figure 1). The rest of his physical examination was unremarkable.

Biopsy of the anorectal lesion was performed. Hematoxylin/eosine stain of tissue showed inflammation and granuloma with necrosis (Figure 2a). Ziehl-Nielsen stain preparation was positive for acid-fast bacillus (Figure 2b).

Patient's CD4 was 176 cells/mm ${ }^{3}$ and HIV-1 RNA was 231.748 (5.36 log) copies. Patient was started on antiretrovirals (ZDV/ 3TC/EFV) and treatment with tuberculostatics was also begun with isoniazid, rifampicin and pyrazinamide. After 30 days, perianal fistula had dramatically reduced. The patient gained weight and had great relief of local pain. By the end of treatment (six months), the lesions were totally healed.

Two hypothesis can explain anal involvement by TB: swallowing of respiratory secretions containing large quantity of Koch's bacillus or following reactivation of a latent focus [1,2,4].

Fistula is the most frequent lesion of anorectal and perianal TB ( $80 \%$ to $91 \%$ of cases). The long duration of perianal lesions is very characteristic, as is recurrence of fistula despite well-managed surgical treatment, which indicates that histological investigations to look for TB on the excised tissue should be conducted in all cases, especially in AIDS patients. The ulcerated form of anal TB usually presents as a superficial ulceration and it may be very painful, or the patient may have only few symptoms [1,6]. Although the incidence of TB is increasing in HIV patients, especially in extra-pulmonary forms, anoperineal region is rarely affected $[1,4]$. 
Anal tuberculosis is less common and has a distinct clinical presentation, when compared to other forms of gastrointestinal TB. Tuberculous fistulae are usually multiple, with pain, local swelling and anal discharge, sometimes purulent. Constitutional symptoms as anorexia, fever, weight loss or malaise usually are not present [2]. Differential diagnosis of anal TB must be done specially with Crohn's disease, due to similar clinical, radiological and endoscopic features [1,7].

Histological examination is mandatory if the patient has had or still has tuberculosis elsewhere in the body [1,6]. Stool cultures are rarely useful and they are not routinely performed $[7,8]$. The typical histological lesion is the epithelioid and giant cell tubercle around a zone of caseous necrosis, but the characteristic caseation is not always present. Diagnosis can also be done by finding acid-fast bacillus in anal lesions by direct examination; culture is also possible. [1,6].

This brief case report depicts an uncommon etiological cause of anal ulceration and perianal discharge in a HIV patient. The authors conclude that the diagnosis of TB has to be considered in the differential diagnosis of anoperineal ulcers and fistulae, especially in AIDS patients, since treatment with antituberculous agents may provide complete recovery [1,4].

\section{Vicente Sperb Antonello, Leonardo Santos Hoff, Darci Kaercher Júnior and Breno Riegel Santos vicente_antonello@hotmail.com}

\section{References}

1. Sultan S., Azria F., Bauer P., Abdelnour M., Atienza P. Anoperineal Tuberculosis: Diagnostic and Management Considerations in Seven Cases. Dis Colon Rectum 2002;45:407-10.

2. Sharma M.P., Bhatia V. Abdominal Tuberculosis. Indian J Med Res 2004;120:305-15.

3. Bromberg S.H., Faroud S., de Castro F.F., Morrone N., de Godoy A.C., França L.C.M. Tuberculose Ileocecal isolada simulando Neoplasia Maligna e Doença de Crohn. Rev Ass Med Brasil 2001;47(2):125-8.

4. Akgun E., Tekin F., Ersin S., Osmanoglu H. Isolated perianal tuberculosis. The Netherlands Journal of Medicine 2005;63(3):115-17.

5. Adhami S., Duthie G., Greenstone M. A Tuberculous Anal Fistula. J R Soc Med 1999;92:467-8.

6. Kraemer M., Gill S.S., Seow-Choen F. Tuberculous Anal Sepsis: Report of Clinical Features in 20 Cases. Dis Colon Rectum 2000;43(11):1589-91.

7. Singhal A., Gulati A., Frizellb R., Manning A.P. Abdominal tuberculosis in Bradford, UK: 1992-2002. European Journal of Gastroenterology \& Hepatology 2005;17:967-71.

8. Marshall J.B. Tuberculosis of the gastrointestinal tract and peritoneum. Am J Gastroenterol 1993;88:989-999. 